ARCHAEOLOGIA AUSTRIACA

Zeitschrift zur Archäologie Europas

Journal on the Archaeology of Europe

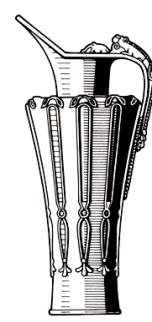

Band 101/2017 



\section{Archaeologia Austriaca}

Zeitschrift zur Archäologie Europas

Journal on the Archaeology of Europe

\section{Band 101/2017}

Herausgeber:

OREA - Institut für Orientalische und Europäische Archäologie Österreichische Akademie der Wissenschaften

Institut für Urgeschichte und Historische Archäologie Universität Wien 
Archaeologia Austriaca erscheint einmal im Jahr und bringt Arbeiten aus dem Gesamtgebiet der europäischen Ur- und Frühgeschichte. Die Zeitschrift umfasst alle Zeitperioden vom Paläolithikum bis in die Neuzeit, die im Rahmen von archäologischen, anthropologischen und allen archäo-interdisziplinären Methoden inklusive der Naturwissenschaften behandelt werden.

Bestellungen sind zu richten an Ihre Buchhandlung oder den Verlag der Österreichischen Akademie der Wissenschaften, Dr. Ignaz Seipel-Platz 2, 1010 Wien, Tel. +43-1-51581/3402-3406, Fax +43-1-51581/3400, http://verlag.oeaw.ac.at,

E-Mail: verlag@oeaw.ac.at

Einreichung von Beiträgen: elektronisch unter http://orea.vlg.oeaw.ac.at/index.php/archaeologiaaustriaca/index

Die Publikationsrichtlinien zur Erstellung des Textes und die Dokumentenvorlage des Verlages der ÖAW finden sich unter

http://www.orea.oeaw.ac.at. Bei Fragen wenden Sie sich an: orea@oeaw.ac.at

Die Autoren und Autorinnen sind für die Bildrechte verantwortlich.

Besprechungsexemplare nimmt das Institut für Orientalische und Europäische Archäologie, Österreichische Akademie der Wissenschaften, Hollandstraße 11-13, 1020 Wien, entgegen, E-Mail: orea@oeaw.ac.at

Als internationale wissenschaftliche peer-reviewed Zeitschrift von der ÖAW gefördert.

Gedruckt mit Unterstützung der Universität Wien, Historisch-Kulturwissenschaftliche Fakultät und der Abteilung Wissenschaft und Forschung der Gruppe Kultur, Wissenschaft und Unterricht des Amtes der Niederösterreichischen Landesregierung.

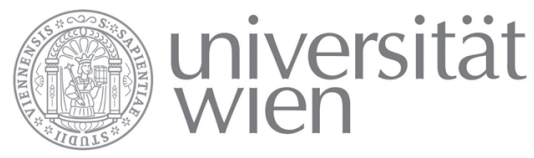

WISSENSCHAFT • FORSCHUNG NIEDERÖSTERREICH

\section{Historisch-Kulturwissen- schaftliche Fakultät}

Bibliografische Information der Deutschen Nationalbibliothek: Die Deutsche Nationalbibliothek verzeichnet diese Publikation in der Deutschen Nationalbibliografie, detaillierte bibliografische Daten sind im Internet über http://dnb.d-nb.de abrufbar.

Herausgeber / Editors:

OREA - Institut für Orientalische und Europäische Archäologie,

Österreichische Akademie der Wissenschaften

Institut für Urgeschichte und Historische Archäologie, Universität Wien

Herausgebergremium / Editorial Board:

Barbara Horejs, Michaela Lochner, Michael Doneus, Timothy Taylor, Claudia Theune

Wissenschaftlicher Beirat / Advisory Board:

Alexandra Busch, Svend Hansen, Peter Pavúk, Ernst Pernicka, Katarina Katja Predovnik, Eva Rosenstock, Stefanie Samida,

Jiří Svoboda, Biba Teržan, Maria Teschler-Nicola

Schriftleitung / Scientific Editor: Estella Weiss-Krejci

Redaktionsassistenz / Copy Editing Assistance: Laura Burkhardt, Barbara Hütthaler, Ulrike Schuh

Buchrezensionen / Book Review Editors: Katharina Rebay-Salisbury, Michaela Zavadil

Englische Textkorrekturen / English Language Editing: Carolyn Aslan, Chris Cumberpatch, Hazel Harrison

Alle Rechte vorbehalten / All rights reserved.

ISBN 978-3-7001-8196-5

ISSN 0003-8008

Copyright (C) 2017 by

Österreichische Akademie der Wissenschaften, Wien / Austrian Academy of Sciences, Vienna

Satz / Layout: María Antonia Negrete Martínez

Druck / Printing and Binding: Prime Rate kft., Budapest

http://epub.oeaw.ac.at/8196-5

http://verlag.oeaw.ac.at

Printed and bound in the EU 